\title{
INVESTIGACIONES
}

\section{Perfil por competencias gerenciales en directivos de instituciones educativas}

\author{
Management skills profile in directors of educational institutions
}

\author{
Nemecio Núñez Rojas, ${ }^{a}$ Doris Díaz Castillo ${ }^{b}$ \\ a'Universidad Católica Santo Toribio de Mogrovejo, Chiclayo, Perú \\ Telf.: (51) 958432417. Correo electrónico: nnunez@usat.edu.pe \\ bDepartamento de Humanidades en la Universidad Católica Santo Toribio de Mogrovejo, Chiclayo, Perú \\ Telf.: (51) 985560092. Correo electrónico: mdiaz@usat.edu.pe
}

El propósito de este estudio es la elaboración y valoración de un perfil por competencias gerenciales para los directivos de las Instituciones Educativas de Educación Básica en el contexto de la mejora continua de la educación sustentada en las normas internacionales y los modelos de calidad. Se utilizó el método de la encuesta para recoger las opiniones y valoraciones de 340 profesionales, entre directivos y profesores que laboran en escuelas urbanas y rurales de tres regiones del norte del país. Las competencias gerenciales que deben tener los directivos de las instituciones educativas son 1) el manejo de dirección, necesaria para el emprendimiento de procesos de gestión escolar académicos y administrativos; 2) el liderazgo, como una actuación de compromiso, iniciativa, creatividad, visión de futuro y sólidos valores en el desempeño personal y profesional; y 3) la gestión del cambio, a través de actividades y proyectos innovadores para alcanzar las metas institucionales.

Palabras clave: competencias gerenciales, gestión educativa, calidad de la educación, competencias profesionales.

\section{ABSTRACT}

The purpose of this study is the development and evaluation of a profile of managerial skills for headmasters of educational institutions of elementary education in the context of the continuous improvement of education based on international standards and quality models. The survey method was used to collect the opinions and assessments of 340 professionals, including managers and teachers that work in rural and urban schools in three regions in the north of the country. Necessary management skills for educational institutions include: 1) management direction, necessary for the undertaking of academic and administrative processes of school management; 2) leadership, as an act of commitment, initiative, creativity, vision and strong values in personal and professional performance, 3) change management, through innovative activities and projects to achieve institutional goals.

Key words: management skills, education management, education quality, professional competence. 


\section{INTRODUCCIÓN}

Gestionar la calidad de la educación es uno de los retos que la sociedad actual demanda a los principales actores que están involucrados en el proceso de acreditación, la mejora continua y la excelencia de las instituciones educativas.

La tendencia es crear una cultura de la calidad que permita mejorar los productos y los servicios, de acuerdo con las normas internacionales de calidad. En el Perú, se tiene formalizado el Sistema Nacional para la Evaluación y Acreditación de la Calidad Educativa, cuya misión es acreditar a las instituciones educativas en nombre del Estado Peruano; para ello, se tiene que haber hecho la autoevaluación y evaluación externa (SINEACE, 2016).

Transitar por la calidad depende de los actores directos, especialmente de los directivos, por ser ellos los que generan las condiciones para el proceso de acreditación de la calidad educativa. Es sin duda un problema complejo, con muchas aristas, pero con oportunidades favorables para aportar investigaciones que generen un impacto práctico en la cultura de la calidad educativa. Este estudio focaliza su atención en las competencias gerenciales que deben tener los directivos de las Instituciones Educativas de Educación Básica, teniendo como base el análisis de las tendencias sobre este tema y las percepciones de los docentes. Se enfatiza en los rasgos que componen el perfil por competencias gerenciales de los directivos, identificados a partir de los problemas del contexto que estos deben resolver y que dan lugar a los desempeños requeridos.

El perfil del directivo comprende las competencias gerenciales: liderazgo, capacidad de dirección y gestión del cambio. La muestra de estudio fue de 340 profesionales que laboran en tres regiones del norte peruano: Lambayeque, Cajamarca y La Libertad, a quienes se aplicó un cuestionario que contiene los desempeños propuestos en las competencias.

\subsection{LAS COMPETENCIAS GERENCIALES EN LA GESTIÓN DE PROCESOS}

Competencia es "un saber integrado derivado de los conocimientos, habilidades y valores que la persona va desarrollando continuamente en su vida, para ser utilizados en su desempeño personal y profesional en los escenarios laborales y sociales en los cuales se desenvuelve" (Núñez et al., 2014, p. 30).

Para Hellriegel, Jackson y Slocum (2002), las competencias gerenciales "son el conjunto de conocimientos, habilidades, comportamientos y actitudes que una persona debe poseer para ser efectiva en un amplio abanico de puestos y en distintas clases de organizaciones" (p. 4). Los gerentes son personas que planean, organizan, dirigen y controlan los capitales y recursos con los que cuentan las organizaciones para alcanzar sus sueños, participan en la construcción del futuro empresarial y de su propio futuro; tienen uno de los retos más difíciles de lograr, que las organizaciones trasciendan y sean reconocidas, lo que permite predecir, que seguramente ellos también trascenderán y serán reconocidos.

Uno de los modelos sobre las competencias gerenciales en el contexto latinoamericano, es el propuesto por Arbaiza (2011), que consiste en un pentágono conformado por las siguientes competencias: 1) Manejo personal: favorecen la efectividad del rendimiento individual en situaciones de presión y dificultad, 2) Manejo de acción: capacidades de organización y planificación de planes de acción; 3) Competencias de manejo de entorno: asociadas a la gestión del entorno laboral; 4) Manejo interpersonal: comprender las 
necesidades propias y las de los demás; y, 5) Manejo de la influencia: deseo de producir un impacto sobre los demás, persuadirlos con el fin de lograr que sigan un plan de acción.

La gestión de procesos es una de las disciplinas de la gestión que contribuye a la dirección de la institución o empresa para identificar, representar, diseñar, formalizar, controlar y hacer más productivos los procesos para lograr la confianza en el usuario o cliente (Bravo, 2013). Un proceso es una competencia de la organización que implica el trabajo en equipo de las personas, en una secuencia organizada de actividades, estructuras y recursos que trasciende las áreas. En el particular caso de las instituciones educativas, siendo estas de gestión públicaestatal o de privados, se tienen que asumir estas prácticas por procesos dado que estos representan el hacer de la organización, del ciclo completo como, por ejemplo: la gestión de los aprendizajes de los estudiantes, el servicio de la capacitación de los profesores, entre otros.

Por tanto, las competencias de las personas para la gestión de procesos son clave. Entre las competencias genéricas propuestas por Bravo (2013) tenemos: "querer el trabajo, trabajar metodológicamente y querer el cambio" (p. 89). El mismo autor identifica competencias del equipo directivo, como "trabajar con visión estratégica y sistémica, trabajar en equipo, desarrollar la visión por procesos, gestionar el cambio y crear ambiente para lograr participación" (p. 90).

\section{MÉTODO}

Esta investigación es de tipo aplicada y es descriptiva, dado que las competencias gerenciales de los directivos fueron sometidas a la valoración de 340 profesionales entre profesores y directivos, de tres regiones del norte del país: Lambayeque, La Libertad y Cajamarca. A decir de Salkind (1999), la investigación descriptiva reseña las características de un fenómeno existente, la encuesta permite que se evalúe la situación actual de cualquier aspecto, es decir, primero obtener una imagen amplia de un fenómeno que nos podría interesar explorar. Cea D'ancona (2010) sostiene que "la encuesta continúa siendo la estrategia más popular en la investigación social” (p. 13).

\subsection{OBJETIVO}

El objetivo principal de este estudio es elaborar y valorar un perfil por competencias gerenciales para los directivos de las instituciones educativas de educación básica en el contexto de la mejora continua de la calidad educativa.

\subsection{POBLACIÓN Y MUESTRA}

La población de docentes en las tres regiones seleccionadas totaliza 71132, de los cuales 26819 son de Cajamarca, 27055 de La Libertad y 17261 de Lambayeque (Ministerio de Educación: ESCALE, 2015). La muestra de estudio la conformaron 340 profesionales de tres regiones del país: Lambayeque, Cajamarca y La Libertad; son profesores seleccionados por el Ministerio de Educación del Perú, y que participaron como representantes de sus regiones en calidad de becarios en la capacitación de "Especialización Invierno" que se realizó en agosto. A ellos se aplicó la encuesta en forma presencial y son profesores con un perfil profesional relevante por sus competencias de desempeño. 


\subsection{INSTRUMENTO}

Se aplicó un cuestionario a los directivos y docentes de tres regiones del norte del país, con la finalidad de recoger sus valoraciones sobre las competencias gerenciales y sus respectivos criterios de desempeños (anexo 1). Su fiabilidad es alta, pues el coeficiente Alfa de Cronbach es de 0.979 y su validez se determinó mediante el KMO cuyo valor es de 0.977 . Metodológicamente responde al enfoque socioformativo de las competencias (Tobón, 2015), pues los 33 ítems que lo conforman se derivan de los problemas del contexto que deben resolver los directivos y, que dieron lugar a las competencias y criterios de desempeños formulados.

\subsection{PROCEDIMIENTO DE RECOGIDA Y ANÁLISIS DE DATOS}

Los datos se recogieron directamente de los profesores y directivos que conformaron la muestra de estudio, aprovechando la concentración de los mismos en un taller de capacitación para profesores que se dio en la Universidad en convenio con el Ministerio de Educación. Luego, se elaboró una base de datos en el SPSS versión 22 y se obtuvo el análisis de fiabilidad y validez, además se elaboraron tablas y gráficos estadísticos, el Análisis de Componentes Principales, el valor de promedio, la varianza y la desviación estándar.

\section{RESULTADOS}

Se realizó la extracción factorial por el Análisis de Componentes Principales y El KMO obtenido es de 0.977. Este método de extracción factorial permite "determinar el número mínimo de factores comunes capaces de reproducir la varianza observada en la matriz de correlaciones inicial" (García, Gil \& Rodríguez, 2000, p. 106). Esta prueba permite averiguar si los ítems presentan características comunes, de modo que se agrupan en componentes que explican la varianza total, y se presentan ordenadamente en función del que mayor cantidad explique.

Tabla 1. Varianza total explicada del Cuestionario

\begin{tabular}{|l|c|c|c|c|c|c|c|c|c|}
\hline \multirow{2}{*}{ Componente } & \multicolumn{3}{|c|}{$\begin{array}{c}\text { Autovalores } \\
\text { iniciales }\end{array}$} & \multicolumn{3}{c|}{$\begin{array}{c}\text { Sumas de extracción de } \\
\text { cargas al cuadrado }\end{array}$} & \multicolumn{3}{c|}{$\begin{array}{c}\text { Sumas de rotación de } \\
\text { cargas al cuadrado }\end{array}$} \\
\cline { 2 - 11 } & Total & $\begin{array}{c}\% \text { de } \\
\text { varianza }\end{array}$ & $\begin{array}{c}\% \\
\text { acumulado }\end{array}$ & Total & $\begin{array}{c}\% \text { de } \\
\text { varianza }\end{array}$ & $\begin{array}{c}\% \\
\text { acumulado }\end{array}$ & Total & $\begin{array}{c}\% \text { de } \\
\text { varianza }\end{array}$ & $\begin{array}{c}\% \\
\text { acumulado }\end{array}$ \\
\hline Ítem_1 & 20,053 & 60,767 & 60,767 & 20,053 & 60,767 & 60,767 & 8,524 & 25,830 & 25,830 \\
\hline Ítem_2 & 1,299 & 3,937 & 64,704 & 1,299 & 3,937 & 64,704 & 8,089 & 24,512 & 50,341 \\
\hline Ítem_3 & 1,093 & 3,311 & 68,015 & 1,093 & 3,311 & 68,015 & 5,832 & 17,673 & 68,015 \\
\hline Ítem_4 &, 811 & 2,459 & 70,473 & & & & & & \\
\hline$\ldots . . .$. & & & & & & & & & \\
\hline Ítem_33 &, 118 &, 359 & 100,000 & & & & & & \\
\hline
\end{tabular}


En la Tabla 1 se pueden evaluar los resultados de la varianza total explicada. Las cifras son las obtenidas con el autovalor 1 , se puede evidenciar que en la columna del $\%$ de la varianza dentro de autovalores iniciales, el primer componente explica un $60,767 \%$ de la varianza total (es el que mejor explica la varianza total), el segundo el 3,937\% de total residual restante y el tercero el 3,311. Es decir, el número total de factores obtenidos es tres, el criterio tomado para dicha decisión es que eligen aquellos que tienen autovalores (cantidad de varianza total explicada por un componente) mayores a la unidad y los cuales explican el 68,015\% de la varianza total.

Tabla 2. Matriz de componentes rotados del cuestionario

\begin{tabular}{|c|c|c|c|}
\hline \multirow{2}{*}{ Ítems } & \multicolumn{3}{|c|}{ Factores } \\
\hline & 1 & 2 & 3 \\
\hline Ítem_1 &, 314 & ,346 & ,685 \\
\hline Ítem_2 & ,349 & ,363 & ,655 \\
\hline Ítem_3 & ,340 & ,345 & 683 \\
\hline Ítem_4 & ,337 & ,321 & ,725 \\
\hline Ítem_5 & ,234 & ,389 & 690 \\
\hline Item_6 & ,451 & ,358 & ,629 \\
\hline Ítem_7 & ,538 & ,306 & ,489 \\
\hline Ítem_8 &, 531 & ,187 & ,503 \\
\hline Ítem_9 & ,634 & ,337 & ,385 \\
\hline Ítem_10 &, 534 & ,397 & ,387 \\
\hline Ítem_11 & ,700 & ,247 & ,224 \\
\hline Ítem_12 & ,628 & ,381 & ,370 \\
\hline Ítem_13 & ,641 & ,190 & ,330 \\
\hline Ítem_14 & ,557 & ,415 & ,330 \\
\hline Ítem_15 & ,736 & , 190 & 290 \\
\hline Ítem_16 & ,526 & ,388 & ,337 \\
\hline Ítem_17 & ,655 & , 458 & ,304 \\
\hline Ítem_18 & ,678 & ,310 & ,235 \\
\hline Ítem_19 & ,551 & ,527 & ,311 \\
\hline Ítem_20 & ,636 & ,468 & ,231 \\
\hline Ítem_21 & ,639 & ,503 & 249 \\
\hline Ítem_22 & ,625 & ,510 & ,346 \\
\hline
\end{tabular}




\begin{tabular}{|c|c|c|c|}
\hline Ítem_23 &, 528 &, 528 &, 417 \\
\hline Ítem_24 &, 365 &, 685 &, 388 \\
\hline Ítem_25 &, 517 &, 634 &, 245 \\
\hline Ítem_26 &, 513 &, 655 &, 175 \\
\hline Ítem_27 &, 504 &, 609 &, 242 \\
\hline Ítem_28 &, 333 &, 682 &, 342 \\
\hline Ítem_29 &, 174 &, 757 &, 341 \\
\hline Ítem_30 &, 288 &, 726 &, 373 \\
\hline Ítem_31 &, 297 &, 709 &, 304 \\
\hline Ítem_32 &, 362 &, 674 &, 349 \\
\hline Ítem_33 &, 315 &, 740 &, 369 \\
\hline
\end{tabular}

Método de extracción: análisis de componentes principales. Método de rotación: Varimax con normalización Kaiser. a. La rotación ha convergido en 9 iteraciones.

Fuente: elaboración propia.

De este análisis se determina que el perfil de los directivos para las instituciones educativas se reduce de cinco a tres competencias. Las tres competencias a las que adelante denominaremos "competencias específicas" son las que reúnen prácticamente a la totalidad de desempeños propuestos; es decir, hay un ajuste en el número de competencias mas no en los desempeños.

La matriz de componentes rotados (Tabla 2), permitió la selección de los ítems finales para la interpretación de la relación entre una variable y un factor, suele considerar como razonable aquellos pesos factoriales ortogonales mayores de 0,30 (aunque hay autores que consideran el 0,40 e incluso el 0,5) (Buendía \& Hidalgo, 2006). En el caso del estudio, teniendo en cuenta que todos los ítems estaban representados en la matriz, se consideró el peso factorial mínimo de los mismos que correspondió al ítem 23 con un valor de 0,528.

En consecuencia, a partir de la distribución de la carga factorial de cada variable en los tres componentes extraídos en la matriz de componentes principales rotada, se pudo determinar las variables que saturan cada componente (con carga superior a 0,528) (Tabla 2). A continuación, se exponen los tres factores que se obtuvieron luego del análisis de componentes principales.

\subsection{FACTORES EXTRAIIDOS DEL CUESTIONARIO}

Tres son los componentes extraídos del Análisis de Componentes Principales (ANP). Los resultados obtenidos (Tabla 2) permitieron el agrupamiento de los ítems, por criterios de desempeños de las competencias. "Las competencias y criterios de desempeño expresan lo que el egresado o el profesional será capaz de resolver al enfrentarse a las situaciones del contexto actual y escenarios futuros, expresado en desempeños observables y con evidencias" (Núñez et al., 2014, p. 255). 
Las competencias: Manejo de Dirección, Gestión Compartida del Cambio y Manejo de Liderazgo, que son las obtenidas en los tres factores del Análisis Factorial, son competencias específicas, inherentes al desempeño de los directivos y gerentes educativos.

\subsubsection{Factor I: Competencia "Manejo de Dirección”}

El Manejo de Dirección, en los directivos es una de las competencias gerenciales que comprende varios desempeños específicos (Tabla 3).

Tabla 3. Carga Factorial de los ítems que saturan el Factor I

\begin{tabular}{|c|c|c|}
\hline Ítem & Enunciados/Desempeños & Carga Factorial \\
\hline 7 & $\begin{array}{l}\text { Dirige la institución educativa de acuerdo con los fundamentos de la } \\
\text { gestión educativa y al contexto. }\end{array}$ &, 538 \\
\hline 8 & Genera Redes educativas de apoyo a la Institución Educativa. &, 531 \\
\hline 9 & $\begin{array}{l}\text { Garantiza la articulación del proyecto educativo institucional con el } \\
\text { entorno }\end{array}$ & ,634 \\
\hline 10 & Informa de manera objetiva a la comunidad los logros y necesidades. &, 534 \\
\hline 11 & $\begin{array}{l}\text { Determina las condiciones para la evaluación y/o selección del per- } \\
\text { sonal. }\end{array}$ & ,700 \\
\hline 12 & $\begin{array}{l}\text { Promueve la participación de los principales actores de la comunidad } \\
\text { educativa en el desarrollo del proyecto educativo }\end{array}$ & ,628 \\
\hline 13 & $\begin{array}{l}\text { Desarrolla y mantiene redes de apoyo institucional para los estudi- } \\
\text { antes dentro de la comunidad educativa. }\end{array}$ & ,641 \\
\hline 14 & $\begin{array}{l}\text { Diseña actividades extracurriculares orientadas a integrar y fortalecer } \\
\text { las relaciones con la comunidad. }\end{array}$ &, 557 \\
\hline 15 & $\begin{array}{l}\text { Establece y mantiene procedimientos de monitoreo y evaluación del } \\
\text { desempeño del personal. }\end{array}$ & ,736 \\
\hline 16 & Gestiona los recursos financieros y físicos. &, 526 \\
\hline 17 & Gestiona la dimensión pedagógica de la organización escolar. & 655 \\
\hline 18 & Administra el talento humano de la institución educativa & ,678 \\
\hline 19 & Participa del trabajo en equipo asumiendo roles y tareas. &, 551 \\
\hline 20 & $\begin{array}{l}\text { Asume tareas, actividades, proyectos que requieren del aporte de dis- } \\
\text { tintos miembros y disciplinas científicas. }\end{array}$ & ,636 \\
\hline 21 & $\begin{array}{l}\text { Es responsable del trabajo en equipo y de la comunicación para el } \\
\text { cumplimiento de las tareas. }\end{array}$ & ,639 \\
\hline 22 & $\begin{array}{l}\text { Contribuye a que el equipo tenga una visión compartida y claros pro- } \\
\text { gramas de trabajo. }\end{array}$ & ,625 \\
\hline
\end{tabular}


La competencia "Manejo de Dirección" en el directivo de una institución educativa implica emprender procesos de gestión escolar académicos y administrativos de una institución educativa, fundamentada en la persona humana, el enfoque integral de la gestión de procesos y los avances científicos y tecnológicos.

En nuestro medio, referirse a la dirección de una institución es asociarla a la jerarquía, dado que es en la "alta dirección" donde se toman las decisiones importantes. "La idea de conducción ligada solo a la noción de jerarquía sucumbe frente a la nueva situación de incertidumbre, quiebres históricos y transformaciones" (Pozner, Ravela \& Fernández, 2000, p. 6).

El direccionamiento de las instituciones educativas requiere de un planeamiento estratégico a mediano y largo plazo, concretado en el proyecto estratégico, en el cual está claramente definida la visión compartida, la misión, los objetivos estratégicos, las estrategias de implementación de la propuesta pedagógica, los procesos de gestión y de innovación. La responsabilidad de los directivos de la institución es elaborar un proyecto de desarrollo institucional participativo, en el cual se encuentren plasmados los intereses, necesidades y expectativas de todos o la gran mayoría de los integrantes de la comunidad educativa, es decir, los implicados internos y externos a la institución.

\subsubsection{Factor II: Competencia "Gestión Compartida del Cambio"}

En la Tabla 4, está la agrupación de ítems que forman parte del Factor II y corresponde a la competencia Gestión Compartida del Cambio.

Tabla 4. Carga Factorial de los ítems que saturan el Factor II

\begin{tabular}{|c|c|c|}
\hline Ítem & Enunciados/Desempeños & Carga Factorial \\
\hline 23 & Tiene un sentido de reto para que el equipo alcance metas. &, 528 \\
\hline 24 & Tiene compromiso ético en el trato con las personas. & ,685 \\
\hline 25 & Genera el cambio colectivo transmitiendo ideas innovadoras. & 634 \\
\hline 26 & $\begin{array}{l}\text { Busca nuevas formas de hacer las cosas basado en su capacidad de } \\
\text { aprendizaje. }\end{array}$ & 655 \\
\hline 27 & $\begin{array}{l}\text { Genera planteamientos y soluciones innovadoras a los problemas de la } \\
\text { gestión escolar. }\end{array}$ & ,609 \\
\hline 28 & $\begin{array}{l}\text { Se comunica en forma oral y escrita, y comprende los diversos mensajes } \\
\text { en una variedad de situaciones. }\end{array}$ & 682 \\
\hline 29 & Controla sus emociones al comunicarse y actúa de manera apropiada. & ,757 \\
\hline 30 & Formula los argumentos propios teniendo en cuenta otros puntos de vista. & ,726 \\
\hline 31 & $\begin{array}{l}\text { Asegura los canales de comunicación e información para la mejor toma } \\
\text { de decisiones. }\end{array}$ & ,709 \\
\hline 32 & Informa de manera objetiva a la comunidad los logros y necesidades. & ,674 \\
\hline 33 & Escucha y atiende a los profesores, estudiantes y padres de familia & ,740 \\
\hline
\end{tabular}


La innovación en la institución se da en la medida que existan las condiciones, especialmente aquellas relacionadas con la satisfacción laboral de cada trabajador en un ambiente de confianza. Sin confianza no hay innovación (Senge, 2005). Los directivos en las instituciones educativas generan un ambiente de confianza en sus trabajadores cuando se comunican con ellos, al delegarles funciones y tareas, reconociendo el talento de cada uno, es decir, haciéndole sentir lo valioso que es para la institución.

En la organización, la necesidad de cambios en el sistema de gestión de calidad debe ser planificada, considerando entre otros aspectos, el propósito del cambio y sus consecuencias potenciales, la disponibilidad de recursos y la asignación de responsabilidades (ISO 9001, 2015).

La gestión del cambio es la habilidad para manejarlo, para asegurar la competitividad y efectividad a un largo plazo (Castro, 2005); plantear abiertamente los conflictos, manejarlos efectivamente en búsqueda de soluciones, para optimizar la calidad de las decisiones y la efectividad de la organización. Esta formulación es un valioso aporte por parte de Castro et al. (2013) de sistematizar las habilidades que requiere un líder en el contexto actual para desempeñarse exitosamente y conducir así a su personal en dirección a los objetivos y metas corporativos.

La competencia es gestiona el cambio educativo mediante actividades y proyectos innovadores para alcanzar las metas institucionales con planeación, objetivos estratégicos definidos, responsabilidad y abordando diferentes contextos en el marco del mejoramiento continuo de la institución educativa.

\subsubsection{Factor III: Competencia "Manejo de Liderazgo"}

El Factor III comprende 6 de los 33 ítems del cuestionario 2. En la Tabla 5, observamos la distribución de los mismos con su respectiva carga factorial.

Tabla 5. Carga Factorial de los ítems que saturan el Factor III: Manejo de Liderazgo

\begin{tabular}{|c|l|c|}
\hline Ítem & \multicolumn{1}{|c|}{ Enunciados/Desempeños } & Carga Factorial \\
\hline 1 & $\begin{array}{l}\text { Posee una visión y unos valores sólidos que permitan obtener la confian- } \\
\text { za de su equipo. }\end{array}$ &, 685 \\
\hline 2 & $\begin{array}{l}\text { Tiene capacidad de análisis e interpretación del entorno, así como de } \\
\text { crear redes y alianzas con otras entidades y actores. }\end{array}$ &, 655 \\
\hline 3 & $\begin{array}{l}\text { Fomenta un ambiente de colaboración y trabajo en equipo con liderazgo } \\
\text { participativo. }\end{array}$ &, 683 \\
\hline 4 & Permanece motivado y enfocado a los objetivos a largo plazo. &, 725 \\
\hline 5 & Comprende y gestiona la diversidad y el conflicto. &, 690 \\
\hline 6 & Motiva y guía al personal para aumentar la efectividad de su trabajo. &, 629 \\
\hline
\end{tabular}


La alta dirección debe demostrar su liderazgo y compromiso con respecto al sistema de gestión de calidad (ISO 9001, 2015), "apoyando a otros roles pertinentes de la organización para demostrar su liderazgo, en la forma en la que aplique a sus áreas de responsabilidad” (p. 13).

En contraste con aquellas teorías que describen a los líderes en términos de tareas y comportamientos del líder orientado a la persona, Rosero y Valcárcel (2013) afirman que las teorías más recientes describen a los líderes en términos de articulación y enfoque de una visión y misión, crean y mantienen una imagen positiva en las mentes de sus seguidores, establecen expectativas desafiantes para estos, muestran confianza y respeto por ellos y se comportan de un modo que refuerza la visión y la misión.

La competencia de liderazgo implica demostrar compromiso, iniciativa creativa, visión de futuro, sólidos valores en el desempeño personal y profesional con la finalidad de propiciar una cultura organizacional de calidad basada en el liderazgo transformativo y en el servicio de calidad.

\subsection{VALORACIÓN DE LOS DIRECTIVOS Y PROFESORES SOBRE LAS COMPETENCIAS GERENCIALES POR REGIONES}

Los 340 docentes encuestados laboran en instituciones de educación básica de las regiones de Lambayeque, La Libertad y Cajamarca. El 68,9\% de docentes cuya institución educativa se encuentra en Lambayeque son titulados como profesor o licenciado en educación, el 89,2\%; en Cajamarca y 66,7\% en la Región La Libertad; es decir, los docentes de las tres regiones, en su mayoría, poseen solo el título profesional y no han realizado estudios de postgrado, lo que demuestra la escasa formación continua que acreditan.

$\mathrm{Al}$ analizar las cifras de los docentes que tienen el grado de maestría, en Lambayeque y La Libertad, representan el 30\%, y solo el 9,7\% de profesores de Cajamarca ostentan este grado académico. Respecto al grado de Doctor, las diferencias son estadísticamente no significativas, pues en las tres regiones, no supera el 2,6\% de profesores con el grado máximo.

Gráfico 1. Valoración promedio en las tres competencias del modelo

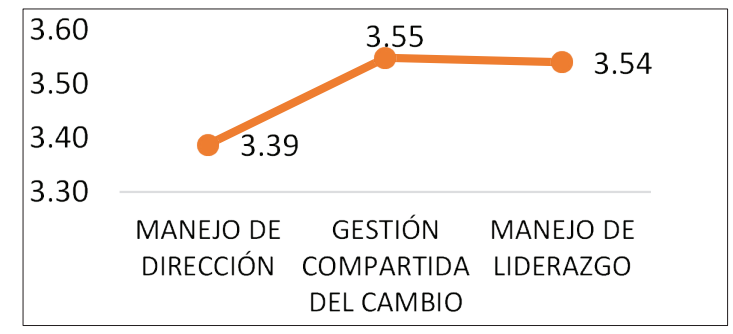

Fuente: elaboración propia.

La valoración que hacen los encuestados es desde la perspectiva: ¿qué competencias gerenciales de los directivos de las Instituciones de Educación Básica debería desarrollarse, dado que son necesarias para una gestión educativa de calidad? Para ello se utilizó una escala de valoración del 1 al 5, en la cual se obtuvieron los siguientes resultados. 
Como se puede apreciar en el Gráfico 1, la competencia con mayor valoración con una Media de 3.55 es la Gestión Compartida del Cambio, seguida muy de cerca por la competencia Manejo de Liderazgo y, con un par de décimas más abajo, la competencia Manejo de Dirección.

Considerando las regiones, en el Gráfico 2, se puede observar que en las tres regiones: Cajamarca, La Libertad y Lambayeque, la mayor valoración promedio está en la competencia de Gestión Compartida del Cambio y corresponde a la Región Cajamarca y es de 3.59; le sigue Manejo de Liderazgo que pertenece a la Región La Libertad con un promedio de 3.57 y, con 3.54, esta misma competencia en la Región Lambayeque. Los menores promedios en las tres regiones son para la competencia Manejo de Dirección.

Gráfico 2. Valoración promedio en las tres competencias del modelo por regiones

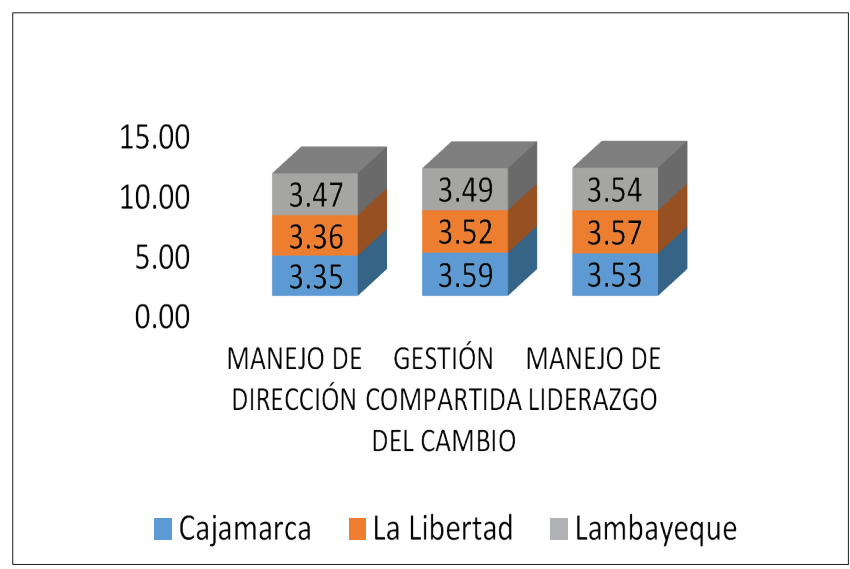

Fuente: elaboración propia.

\section{DISCUSIÓN Y CONCLUSIONES}

Comparar los resultados obtenidos en esta investigación con aquellos obtenidos en otros estudios similares, nos permite sostener que las competencias gerenciales para los directivos de las instituciones de educación básica son diversas, pero que tienen puntos de coincidencia en lo que respecta al liderazgo, manejo de personal, manejo interpersonal, entre otras.

Las aportaciones de los encuestados coinciden con la propuesta realizada por Castro (2005), que consiste en una agrupación de competencias gerenciales: gerenciamiento de la motivación del personal, conducción de grupos de trabajo, liderazgo, comunicación eficaz, dirección de personas, gestión del cambio y desarrollo de la organización.

La competencia Gestión Compartida del cambio, con mayor valoración en la Región Cajamarca (en nuestros resultados), es una competencia que debería ser desarrollada en los directivos. Este dato coincide con el estudio realizado por Palomo (2008), que la ubica en el puesto cinco de seis que comprende las competencias en investigación. En nuestro estudio se ubica en el lugar dos de tres competencias. 
Medina y otros (2012) en una investigación similar sostienen que las competencias gerenciales de primer nivel en una estructura piramidal son el liderazgo, la toma de decisiones, la iniciativa de innovación, el trabajo colaborativo y el desarrollo de las personas; todas estas con un nivel de incidencia mayor a 0.80 en el coeficiente Chi Cuadrado de Pearson. Además, afirman que "la identificación y evaluación de las competencias gerenciales en directivos a nivel organizacional debe ser resultado de un proceso en el que intervengan directivos y trabajadores de diferentes niveles en su conceptualización, selección y valoración en la práctica" (p. 97).

En otro estudio realizado con directores y subdirectores de Instituciones de Educación Básica en Venezuela, los resultados obtenidos son similares a los nuestros, aunque con algunas variantes respecto a las denominaciones de las competencias gerenciales. Los resultados evidencian que, según la percepción de los gerentes educativos encuestados, ellos poseen las competencias personales en un alto dominio de las mismas, especialmente el conocimiento de sí mismo, autorrealización y autocontrol. Igualmente, para las competencias gerenciales en los roles de director, mentor y facilitador con promedios por indicador que oscilan desde 2.98 hasta 3.44 y los ubican en la categoría de alto dominio de la competencia; sin embargo, para el rol de innovador con los indicadores convivir con el cambio (1.66) y pensamiento creativo (1.77) se ubican en muy bajo dominio (Marcano \& Finol de Franco, 2007)

Aristimuño, Guaita y Rodríguez (2010), a partir de los resultados obtenidos en opinión de los docentes encuestados, que en algún momento desempeñaron algún cargo directivo en la institución educativa de educación superior, concluyen que

resulta conveniente destacar que las competencias gerenciales que se presentaron, para su validación, ante los académicos objeto de estudio, se agruparon de acuerdo a su naturaleza, es decir, son de carácter personal (autoconfianza, sensibilidad interpersonal, control de cambio emocional, empatía); organizacional (desarrollo de personas, desarrollo y cambio organizacional, identificación con la organización) y gerencial (trabajo en equipo, liderazgo, pensamiento estratégico, desarrollo de personal). (p. 13).

Con lo anteriormente expuesto y discutido, los resultados obtenidos en este estudio nos permiten asumir que las competencias gerenciales de los directivos van variando en la medida que cambian las condiciones del contexto socio-educativo; sin embargo, es posible determinar algunas invariantes en los desempeños requeridos en el perfil de los directivos. En la actualidad, el gerente educativo debe buscar la participación activa de los docentes, padres y representantes, y miembros de la comunidad, centrar su atención en las necesidades que la institución presenta, encontrar seguridad y protección en sus miembros, pasando desde un inicial involucramiento emocional de las personas entre sí hacia un involucramiento de tipo colectivo.

La problemática de la formación y capacitación de los directivos agudiza las condiciones de la gestión en las Instituciones de Educación Básica. En el Perú, quienes dirigen las escuelas son profesores de aula que fueron formados para la docencia, pero que luego asumieron funciones directivas. Aproximadamente, la mitad de los profesores no han realizado estudios de formación continua como maestrías y doctorados u otras especializaciones similares, y, de los que los han realizado, solo una minoría tiene estudios relacionados con la gestión educativa o la gerencia educativa estratégica. 
Las competencias gerenciales que deben tener los directivos de las instituciones educativas de educación básica son 1) el manejo de dirección, necesaria para el emprendimiento de procesos de gestión escolar académicos y administrativos; 2) el liderazgo, como una actuación de compromiso, iniciativa, creatividad, visión de futuro y sólidos valores en el desempeño personal y profesional, 3) la gestión del cambio, a través de actividades y proyectos innovadores para alcanzar las metas institucionales con planeación, objetivos estratégicos definidos, responsabilidad y abordando diferentes contextos en el marco del mejoramiento continuo de la institución educativa.

\section{REFERENCIAS BIBLIOGRÁFICAS}

Arbaiza, L. (2011). Desarrollo de competencias gerenciales: un modelo alternativo. Buenos Aires: Cengage Learning, Mx-Cengage.

Aristimuño, M., Guaita, W., \& Rodríguez, C. (2010). Las competencias gerenciales en la gestión de instituciones de educación superior. 8th Latin American and Caribbean Conference for Engineering ansd Technology, Arequipa, Perú. 1-2. Recuperado desde http://www.laccei.org/ LACCEI2010-Peru/Papers/Abstracts.\%20pdf/ACC061_Aristimuno_EA.pdf

Bravo, J. (2013). Gestión de Procesos. Santiago de Chile: Editorial Evolución S.A.

Buendía, L., \& Hidalgo, V. (2006). La mejora de un centro multicultural de la Ciudad Autónoma de Ceuta. Revista de Investigación Educativa, 24(1), 261-280. doi:10.6018 / rie.24.1.97691

Castro, F. (2005). Competencias o (Habilidades) Gerenciales: Una herramienta indispensable para el desarrollo organizacional. En L. Marchant (Ed.), Actualizaciones para el Desarrollo Organizacional (pp. 62-68). Chile: Universidad Viña del Mar.

Castro, L., Fossi, L., Guerrero, W., \& Vera, L. (2013). Competencias gerenciales y gestión de los proyectos educativos. Venezuela. Orbis Revista Científica Electrónica de Ciencias Humanas, 9(25), 91-108.

Cea D’Ancona, A. (2010). Métodos de Encuesta. Teoría y Práctica, Errores y Mejora. Madrid: Editorial Síntesis.

García, E., Gil, J., \& Rodríguez, G. (2000). Análisis factorial. Madrid: La Muralla.

Hellriegel, D., Jackson, S., \& Slocum, J. (2002). Administración: Un Enfoque Basado en Competencias (9a ed.). México: Thomson Editores.

ISO 9001. (2015). Sistema de Gestión de Calidad-Requisitos. Suiza: ISO.

Marcano, N., \& Finol de Franco, M. (2007). Competencias personales y gerenciales de los directores y subdirectores de las escuelas básicas. Revista Venezolana de Gerencia, 12(39), 410-430.

Medina, M., Armenteros, M., Guerrero, L., \& Barquero, J.D. (2012). Las Competencias Gerenciales desde una visión estratégica de las organizaciones: un procedimiento para su identificación y evaluación del desempeño. Revista International Administración \& Finanzas, 5(2), 79-100.

Ministerio de Educación del Perú-ESCALE. (2015). Magnitudes de la Educación en el Perú. Lima: MINEDU. Recuperado desde http://escale.minedu.gob.pe/magnitudes

Núñez, N., Vigo, O., Palacios, P., \& Arnao, M. (2014). Formación Universitaria Basada en Competencias: Currículo, Estrategias Didácticas y Evaluación. Chiclayo: USAT.

Palomo, M.T. (2008). El perfil competencial del puesto de director/a de marketing en organizaciones de la Comunidad de Madrid. España: ESIC Editorial.

Pozner, P., Ravela, P., \& Fernández, T. (2000). Diez módulos destinados a los responsables de los procesos de transformación educativa. Buenos Aires: IIPE.

Rosero, L., \& Valcárcel, M. (2013). Liderazgo transformacional y gerencia humanista. Colombia: Universidad EAN, Facultad de Administración, Finanzas y Ciencias Económicas.

Salkind, N. (1999). Métodos de Investigación. México, D.F.: Pearson Educación. 
Senge, P. (2005). Quinta disciplina: El arte y la práctica de la organización abierta al aprendizaje (2da ed.). Buenos Aires: Editorial Granica S.A.

SINEACE. (2016). Modelo de Acreditación para Programas de Educación Superior Universitaria. Lima: Sistema Nacional de Evaluación. Acreditación y Certificación de la Calidad EducativaSINEACE.

Tobón, S. (2015). Formación integral por competencias. Bogotá: ECOE.

Anexo 1: Cuestionario para la Valoración de las Competencias Gerenciales

Indique por favor: El nivel en que cree que la competencia debería desarrollarse en los Directivos de las Instituciones Educativas de Educación Básica.

\section{Competencia: Manejo de Liderazgo}

Demuestra compromiso, iniciativa creativa, visión de futuro, sólidos valores en el desempeño personal y profesional, con la finalidad de propiciar una cultura organizacional de calidad basada en el liderazgo transformativo y en el servicio de calidad.

1. Posee una visión y valores sólidos que permitan obtener la confianza de su equipo y de los grupos de interés de la I.E.

2. Tiene capacidad de análisis e interpretación del entorno, asî como de crear redes y alianzas con otras entidades y actores.

3. Fomenta un ambiente de colaboración y trabajo en equipo a través de un estilo de liderazgo participativo.

4. Permanece motivado y enfocado a los objetivos a largo plazo a pesar de los fracasos o impedimentos en el camino

5. Comprende y gestiona la diversidad y el conflicto, y encuentra espacios de entendimiento y progreso en ellos

6. Motiva y guía al personal para aumentar la efectividad de su trabajo en la Institución Educativa

\begin{tabular}{|l|l|l|l|l|l|}
\hline 1 & 2 & 3 & 4 & 5 \\
\hline 1 & 2 & 3 & 4 & 5 \\
\hline 1 & 2 & 3 & 4 & 5 \\
\hline 1 & 2 & 3 & 4 & 5 \\
\hline 1 & 2 & 3 & 4 & 5 \\
\hline
\end{tabular}

\section{Competencia Manejo de Dirección}

Emprende procesos de gestión escolar académicos y administrativos de una institución educativa, fundamentada en la persona humana, el enfoque integral de la gestión y los avances científicos y tecnológicos.

7. Dirige la I.E. de acuerdo con los fundamentos de la gestión educativa y al contexto de los estudiantes.

8. Genera Redes educativas de apoyo a la I.E para potenciar el proyecto educativo institucional y desarrollar los objetivos estratégicos

9. Garantiza la articulación PEI con las características y necesidades del entorno

\begin{tabular}{|l|l|l|l|l|l|}
\hline & 1 & 2 & 3 & 4 & 5 \\
\hline 1 & 2 & 3 & 4 & 5 \\
\hline
\end{tabular}




\begin{tabular}{|c|c|c|c|c|c|}
\hline $\begin{array}{l}\text { 10. Informa de manera objetiva a la comunidad los logros y necesidades } \\
\text { de la I.E. }\end{array}$ & 1 & 2 & 3 & 4 & 5 \\
\hline $\begin{array}{l}\text { 11. Determina las condiciones para la evaluación y/o selección del } \\
\text { personal de la I.E. }\end{array}$ & 1 & 2 & 3 & 4 & 5 \\
\hline $\begin{array}{l}\text { 12. Promueve la participación de los principales actores de la comunidad } \\
\text { educativa en el desarrollo del PEI }\end{array}$ & 1 & 2 & 3 & 4 & 5 \\
\hline $\begin{array}{l}\text { 13. Desarrolla y mantiene redes de apoyo institucional para los estudiantes } \\
\text { dentro de la comunidad educativa }\end{array}$ & 1 & 2 & 3 & 4 & 5 \\
\hline $\begin{array}{l}\text { 14. Diseña actividades extracurriculares orientadas a integrar y fortalecer } \\
\text { las relaciones con la comunidad }\end{array}$ & 1 & 2 & 3 & 4 & 5 \\
\hline $\begin{array}{l}\text { 15. Establece y mantiene procedimientos de monitoreo y evaluación del } \\
\text { desempeño del personal de la I.E. }\end{array}$ & 1 & 2 & 3 & 4 & 5 \\
\hline $\begin{array}{l}\text { 16. Gestiona los recursos financieros y físicos necesarios para el desarrollo } \\
\text { del servicio educativo }\end{array}$ & 1 & 2 & 3 & 4 & 5 \\
\hline $\begin{array}{l}\text { 17. Gestiona la dimensión pedagógica de la organización escolar, } \\
\text { considerando el desarrollo de capacidades del ser humano. }\end{array}$ & 1 & 2 & 3 & 4 & 5 \\
\hline $\begin{array}{l}\text { 18. Administra el talento humano de la institución educativa de acuerdo } \\
\text { con la normatividad vigente, sus competencias asignadas e indicadores } \\
\text { de gestión }\end{array}$ & 1 & 2 & 3 & 4 & 5 \\
\hline
\end{tabular}

\section{Competencia Trabajo en Equipo}

Demuestra idoneidad en el trabajo en equipo para generar propuestas educativas innovadoras, que contribuyan a la solución de problemas educativos del contexto con el apoyo de otros, de especialistas en distintas áreas de la institución.

19. Participa del trabajo en equipo asumiendo roles y tareas con responsabilidad y resultados concretos como proyectos, informes

20. Asume tareas, actividades, proyectos que requieren del aporte de distintos miembros, disciplinas científicas, aportando lo suyo desde su formación

21. Es responsable del trabajo en equipo, de la comunicación que existe en el mismo para el cumplimiento de las tareas

22. Contribuye a que el equipo tenga una visión compartida y claros programas de trabajo, participando en el análisis y resolución creativa de problemas

23. Tiene un sentido de reto para que el equipo alcance metas cada vez más elevadas, acorde con la visión de la institución educativa

24. Tiene compromiso ético en el trato con las personas considerando los valores universales

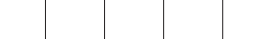




\section{Competencia Gestión del Cambio}

Gestiona el cambio educativo a través de actividades y proyectos innovadores para alcanzar las metas institucionales, con planeación, objetivos estratégicos definidos, responsabilidad y abordando diferentes contextos en el marco del mejoramiento continuo de la institución educativa.

25. Genera el cambio colectivo a través de habilidades de comunicación e interrelación, transmitiendo ideas innovadoras de manera efectiva

26. Busca nuevas formas de hacer las cosas basado en su capacidad de aprendizaje, experimentación y desarrollo de soluciones innovadoras y coherentes con el entorno

27. Genera planteamientos y soluciones innovadoras a los problemas que se le presentan en la gestión escolar

\begin{tabular}{|l|l|l|l|l|l|}
\hline 1 & 2 & 3 & 4 & 5 \\
\hline & 1 & 2 & 3 & 4 & 5 \\
\hline
\end{tabular}

\section{Competencia Capacidad de Comunicación}

Comunicarse con entendimiento de manera verbal, escrita y gestual con los integrantes de la comunidad educativa generando sinergias internas y externas que contribuyan al desarrollo institucional

28. Se comunicación en forma oral y escrita, y comprende los diversos mensajes en una variedad de situaciones que se presentan en la gestión

29. Controla sus emociones al comunicarse y actúa de manera apropiada ante distintas personas y situaciones

30. Formula los argumentos propios, de forma hablada o escrita teniendo en cuenta otros puntos de vista de los integrantes de la comunidad educativa

31. Asegura los canales de comunicación e información para la mejor toma de decisiones

32. Informa de manera objetiva a la comunidad los logros y necesidades de la I.E.

33. Escucha y atiende a las inquietudes, necesidades y expectativas de los profesores, estudiantes y padres de familia

\begin{tabular}{|l|l|l|l|l|l|}
\hline 1 & 2 & 3 & 4 & 5 \\
\hline 1 & 2 & 3 & 4 & 5 \\
\hline 1 & 2 & 3 & 4 & 5 \\
\hline 1 & 2 & 3 & 4 & 5 \\
\hline 1 & 2 & 3 & 4 & 5 \\
\hline
\end{tabular}

